\title{
The remarkable catalytic activity of the saturated metal organic framework V-MIL-47 in the cyclohexene oxidation $\dagger$
}

\author{
Karen Leus, ${ }^{a}$ Ilke Muylaert, ${ }^{a}{ }^{b}$ Matthias Vandichel, ${ }^{b}$ Guy B. Marin, ${ }^{c}$ Michel Waroquier, ${ }^{b}$ \\ Veronique Van Speybroeck ${ }^{* b}$ and Pascal Van Der Voort*a
}

Received 20th May 2010, Accepted 4th June 2010

First published as an Advance Article on the web 18th June 2010

DOI: $10.1039 / \mathrm{c0cc01506g}$

The remarkable catalytic activity of the saturated metal organic framework MIL-47 in the epoxidation of cyclohexene is elucidated by means of both experimental results and theoretical calculations.

Metal organic frameworks (MOFs) are crystalline porous solids composed of a three-dimensional network of metal ions held in place by multidentate organic molecules. ${ }^{1,2}$ Recently, the catalytic applications of MOFs have been reviewed by Hupp et al. and Farrusseng et al. ${ }^{3,4}$ However, catalytic reaction mechanisms in metal organic frameworks and in particular at saturated coordinated centres are still poorly understood.

In this study, we describe the high catalytic activity of a coordinative saturated V-MOF in the liquid phase oxidation of cyclohexene. The metal organic framework MIL-47 consists of a porous terephthalate framework built from infinite chains of $\mathrm{V}^{4+} \mathrm{O}_{6}$ octahedra and has a three-dimensional orthorhombic structure that exhibits large pores. ${ }^{5}$ Every structure unit in MIL-47 contains an $\mathrm{O}-\mathrm{V}^{+\mathrm{IV}}-\mathrm{O}$ bridge. The linkage of the $\mathrm{V}^{+\mathrm{IV}}$ to dicarboxylate groups of the terephthalate linkers results in an octahedral structure unit. Hence each vanadium(+IV) centre is saturated.

The selective oxidation of cyclohexene is particularly interesting as it can yield, depending on the catalyst and the reaction conditions, several products as shown in Scheme 1. In this study, the reaction was carried out with tert-butyl hydroperoxide (70 wt $\%$ TBHP in water) as oxidant and chloroform as solvent. The internal standard used was 1,2,4-trichlorobenzene. The molar ratios were chloroform: cyclohexene:TBHP:1,2,4-trichlorobenzene = 462:1:2:1. The reaction temperature was kept constant at $323 \mathrm{~K}$. Samples were taken out from the reaction mixture at certain times and analyzed by gas chromatography. Next, MIL-47 was compared with a homogeneous vanadium catalyst vanadyl acetylacetonate $\mathrm{VO}(\mathrm{acac})_{2}$. Prior to the reaction, all the catalysts were dried under vacuum. Blank tests were performed without catalyst and no cyclohexene conversion was observed.

\footnotetext{
${ }^{a}$ Department of Inorganic and Physical Chemistry,

Centre for Ordered Materials, Organometallics and Catalysis,

Ghent University, Krijgslaan 281-S3, 9000 Ghent, Belgium.

E-mail: pascal.vandervoort@ugent.be

${ }^{b}$ Centre for Molecular Modeling, Ghent University,

Technologiepark 903, 9052 Zwijnaarde, Belgium.

E-mail:Veronique.vanspeybroeck@ugent.be

${ }^{c}$ Laboratory for Chemical Technology, Ghent University,

Krijgslaan 281-S5, 9000 Ghent, Belgium

$\dagger$ Electronic supplementary information (ESI) available: XRF analysis, nitrogen adsorption isotherms, XRD patterns, experimental and computational details. See DOI: 10.1039/c0cc01506g
}

Fig. 1 depicts the cyclohexene conversion and the detailed product distributions for MIL-47 and the homogeneous $\mathrm{VO}(\mathrm{acac})_{2}$ catalyst. In both reactions, we used an identical amount of V-sites (0.4 mmol, $0.095 \mathrm{~g}$ MIL-47 and $0.106 \mathrm{~g}$ $\left.\mathrm{VO}(\mathrm{acac})_{2}\right)$. The four main reaction products formed are cyclohexene oxide $\mathbf{2}$, cyclohexane-1,2-diol 3, tert-butyl-2cyclohexenyl-1-peroxide $\mathbf{4}$ and 2-cyclohexen-1-one 5. From these figures it can be seen that the homogeneous catalyst $\mathrm{VO}(\mathrm{acac})_{2}$ and the MIL-47 show high reaction rates for the conversion of cyclohexene. The initial turnover frequencies considered at the first $20 \mathrm{~min}$ are $57 \mathrm{~h}^{-1}$ and $43 \mathrm{~h}^{-1}$ for the $\mathrm{VO}(\mathrm{acac})_{2}$ and the MIL-47 respectively. Both catalysts showed an almost linear conversion rate during the first $2 \mathrm{~h}$ of reaction and finally topped out at $60 \%$ conversion. The turnover numbers calculated at the end of the reaction were 62 and 68 for $\mathrm{VO}(\mathrm{acac})_{2}$ and MIL-47 respectively. Moreover, considering the product distributions, truly interesting similarities between both catalysts are observed.

Firstly, the cyclohexene oxide is formed during the first $2 \mathrm{~h}$ and is topped out at $15 \%$, but its yield decreases with increasing reaction time. At the same time and at the same reaction rate the oxide is decreasing, the cyclohexane-1,2-diol formation is observed, indicating a consecutive reaction is taking place. After $5 \mathrm{~h}$, the diol formation reaches a plateau at $15 \%$. Additionally, both catalysts produce a significant amount of tert-butyl-2-cyclohexenyl-1-peroxide $4(30 \%)$ as determined by GC-GC-TOF-MS and a minimal amount of 2-cyclohexen-1-one 5 (5\%).

In order to examine the heterogeneity of the catalytic reaction, a hot filtration experiment was performed. The catalyst was separated from the reaction mixture after $1 \mathrm{~h}$ using a combined nylon-membrane filter. Fig. 2 presents the conversion curve of cyclohexene in the presence of MIL-47 and the cyclohexene conversion after filtration. Also the sum of products 2, 3 and $\mathbf{5}$ is shown with and without the hot filtration. It is clear from this figure that both the conversion of cyclohexene and the formation of oxidized products are strongly reduced after filtration of the catalyst. This indicates that the catalytic reaction is predominantly heterogeneous. There is however some homogeneous catalytic activity left. This activity is due to a combination of radical mechanisms
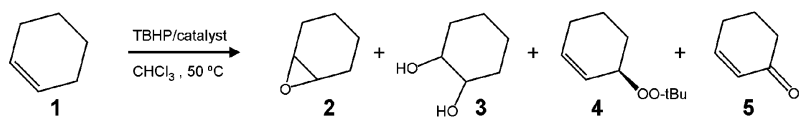

Scheme 1 

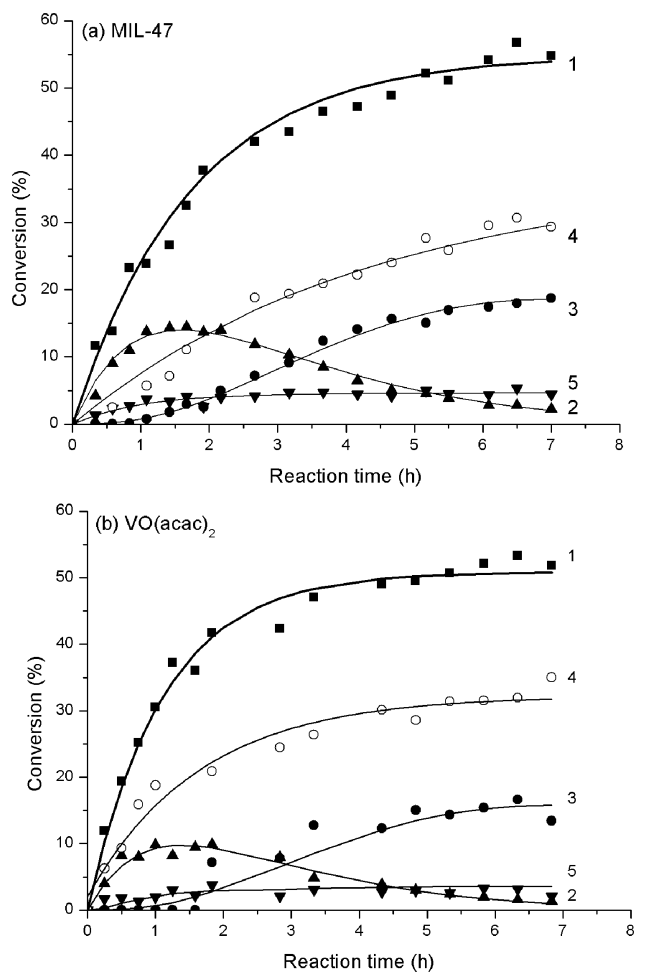

Fig. 1 Total cyclohexene conversion and detailed product distribution in the cyclohexene oxidation with (a) MIL-47 and (b) $\mathrm{VO}(\mathrm{acac})_{2}$ in chloroform with tert-butyl hydroperoxide ( $70 \mathrm{wt} \%$ solution in water) at $323 \mathrm{~K}$. The numbers of the curves correspond with the numbers in Scheme 1.

(formation of product $\mathbf{4}$ occurs via radical pathways (vide infra)) and leached V. By XRF analysis we have determined that $12.8 \%$ of the $\mathrm{V}$-sites have leached out after $1 \mathrm{~h}$ of reaction (see Fig. S.1 in the supplementary information $\dagger$ ). Finally, we also show the catalytic conversion caused by dissolving exactly the amount of $\mathrm{VO}(\mathrm{acac})_{2}$ that corresponds to the $12.8 \%$ leached $\mathrm{V}$-sites $(\boldsymbol{\nabla})$. This leads however to only $5 \%$ conversion.

Furthermore, the stability and the regenerability in terms of morphology and catalytic performance of MIL-47 were studied. The original synthesized MOF exhibits a specific surface area around $800 \mathrm{~m}^{2} \mathrm{~g}^{-1}$. After the first catalytic run, the pores are

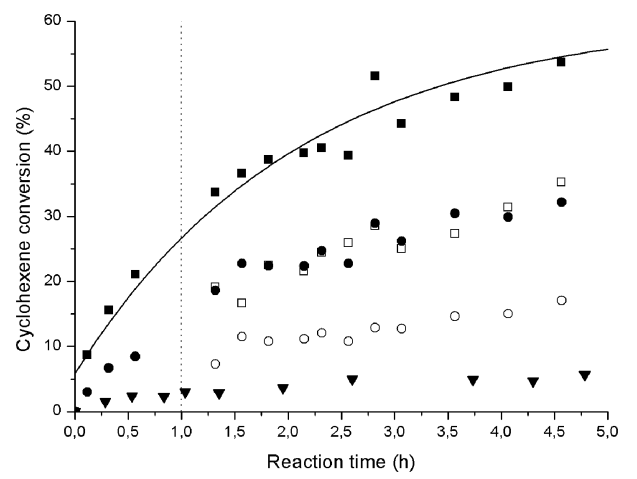

Fig. 2 Hot filtration experiment. Cyclohexene conversion ( $\mathbf{a}$ ) and yield of oxidized products $(\mathbf{O})$ as a function of time. Cyclohexene conversion $(\square)$ and yield of oxidized products $(\bigcirc)$ after filtration of the catalyst. The catalytic activity of $\mathrm{VO}(\mathrm{acac})_{2}$ corresponding to the $12.8 \%$ leached V-sites $(\boldsymbol{\nabla})$. completely clogged by organic compounds. Subsequently, MIL-47 was regenerated by a treatment in a tubular furnace under a nitrogen flow at $523 \mathrm{~K}$ for four hours to remove the organic compounds.

After this calcination treatment the porosity was completely regenerated as can be seen in Fig. S.2.† Additionally, X-ray diffraction (XRD) patterns show that the crystalline structure of MIL-47 is preserved after regeneration (see Fig. S.3†). Finally, the catalytic activity of MIL-47 is tested in a second catalytic run, in which $27 \mathrm{mg}$ catalyst was recovered and used. In order to make a fair comparison, we also show first runs, using respectively $20 \mathrm{mg}$ and $40 \mathrm{mg}$ catalyst, in Fig. 3. Although the kinetics are initially different, the final conversion in the second run does not show any significant loss of activity.

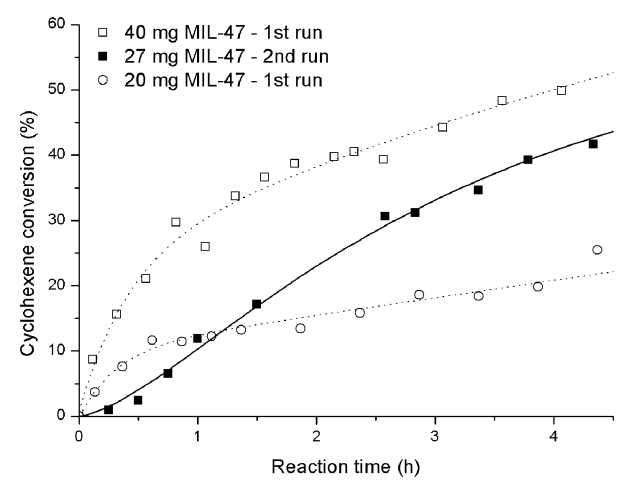

Fig. 3 Cyclohexene conversion for MIL-47 in its first run with ( $\square$ ) $40 \mathrm{mg}$ MIL-47, (○) $20 \mathrm{mg}$ MIL-47 catalyst mass and (ם) after regeneration with $27 \mathrm{mg}$ MIL-47.

Besides MIL-47, two other V catalysts are tested for the oxidation of cyclohexene, namely the supported $\mathrm{VO}_{x} / \mathrm{SiO}_{2}$ and the microporous crystalline VAPO-5. The latter is a crystalline AlPO zeotype, containing vanadium ( + IV) centres. We observed that VAPO-5 is completely catalytically inactive for the oxidation of cyclohexene. This observation is consistent with other research, outlining that VAPO-5 (and more generally MeAPOs) are not suitable as catalysts for liquid phase oxidation reactions. $^{6}$

The supported $\mathrm{VO}_{x} / \mathrm{SiO}_{2}$ catalyst shows a good conversion in a first run (see Fig. S. $4 \dagger$ ). However, after the first catalytic run, $40 \%$ of the vanadium was leached from the silica supported catalyst and no cyclohexene conversion was observed in its second run, indicating the reaction occurs basically homogeneously.

To get more insight into the possible reaction mechanisms and intermediates, theoretical calculations were performed on the $\mathrm{VO}(\mathrm{acac})_{2}$ catalyst, which showed a remarkable resemblance to the MIL-47 catalyst from the experimental results. A comparison of the optimized geometries of the $\mathrm{VO}(\mathrm{acac})_{2}$ system with the XRD data of the MIL-47 indicates that the surroundings of the vanadium atom are very similar geometrically wise (Fig. 4). The initial coordination of TBHP with vanadium is expected to occur in a similar fashion apart from steric constraints. More information on the computational details can be found in the supporting information. $\dagger$ The formation of the epoxide, the diol and the peroxide will 

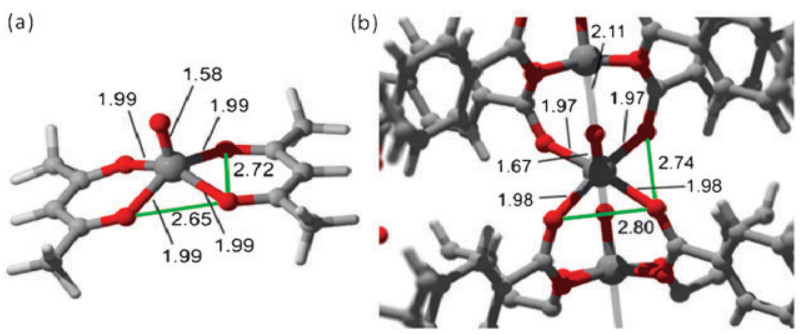

Fig. 4 Comparison of distances (in $\AA$ ) for (a) the homogeneous $\mathrm{VO}(\mathrm{acac})_{2}$ system and (b) the metal organic framework MIL-47.

now be discussed. Because the ketone is not present in large quantities, this reaction pathway will not be considered here.

Fig. 5 shows the epoxidation pathway with $\mathrm{VO}(\text { acac })_{2}$ with the final regeneration step of the catalyst included. The reaction cycle starts with an activation step (A) in which TBHP coordinates with $\mathrm{VO}(\mathrm{acac})_{2}$. Here, an alkylperoxo species, namely $\mathrm{VO}(\mathrm{acac})(\mathrm{OOtBu})$, is formed. All attempts to model an epoxidation cycle on a complex with two acac ligands failed, which suggests that first vacant coordination sites need to be generated, in accordance with earlier literature data on the activity of peroxo and alkylperoxo species for oxidations. ${ }^{7}$ In a second step the actual epoxidation $(\mathbf{E})$ towards cyclohexene oxide can occur easily with an activation barrier of $55.9 \mathrm{~kJ} \mathrm{~mol}^{-1}$ calculated at the B3LYP/6-311+g(3df,2p) level of theory. This step is irreversible which can be deduced from the reverse barrier which is very high $\left(236.8 \mathrm{~kJ} \mathrm{~mol}^{-1}\right)$. After epoxidation, Hacac can coordinate back to the vanadium centre and cyclohexene oxide is split off $(\mathbf{P})$. Notice that a similar ligand exchange with cyclohexene oxide could also occur with other species present in the reaction medium $\left(\mathrm{tBuOH}, \mathrm{H}_{2} \mathrm{O}, \mathrm{TBHP}\right)$. The final regeneration step occurs fast via a proton transfer reaction. Both activation $(\mathbf{A})$ and production $(\mathbf{P})$ are endothermic from our calculations but the equilibrium could significantly shift on inclusion of a larger molecular environment.

In the case of the $\mathrm{VO}(\mathrm{acac})_{2}$ catalysts substantial amounts of acetic acids are formed upon decomposition of Hacac with TBHP. ${ }^{8}$ The subsequent formation of the cyclohexane-1,2-diol can be explained by opening of the epoxide ring by an acid catalyzed hydrolysis mechanism. ${ }^{9}$ In the MIL-47, a small fraction of terephthalic acid is anticipated to catalyze the opening of the epoxide ring.

During the first catalytic cycle, a majority of the reaction product is tert-butyl-2-cyclohexenyl-1-peroxide. This product is formed due to a radical reaction pathway between the substrate and the oxidant TBHP and has been recently observed for unsaturated Co-MOFs. ${ }^{10}$ Freely diffusing peroxy radicals can be generated by a variety of mechanisms in which the oxidation state of vanadium changes.

Similar reaction pathways in the MIL-47 system can be considered, provided one terephthalic acid ligand folds away from the vanadium centre to create a vacancy for the coordination of a TBHP molecule. Subsequently, the actual epoxidation can occur. Further theoretical modelling is in progress to fully investigate the proposed reactions in the MIL-47.

In conclusion, the saturated metal organic framework MIL-47 is catalytically active in the oxidation of cyclohexene,

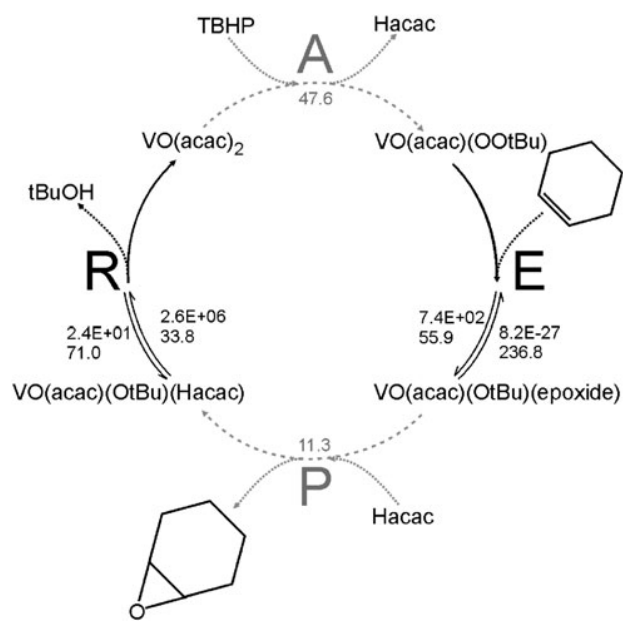

Fig. $5 \mathrm{VO}(\mathrm{acac})_{2}$ epoxidation cycle. Gray numbers indicate reaction energies $\left(\mathrm{kJ} \mathrm{mol}^{-1}\right)$, while black numbers indicate reaction barriers (bottom, $\mathrm{kJ} \mathrm{mol}^{-1}$ ) and reaction rates (top, $\mathrm{s}^{-1}$ ).

showing a similar catalytic activity and product distribution to the homogeneous vanadium complex $\mathrm{VO}(\mathrm{acac})_{2}$. Hot filtration experiments show that the oxidation catalysis occurs predominantly heterogeneously. MIL-47 can be regenerated and reused. Theoretical calculations on the $\mathrm{VO}(\mathrm{acac})_{2}$ system give insights into the reaction pathways towards the reaction products and reveal that the successful epoxidation is accompanied by a linker exchange with TBHP. A similar mobility of the terephthalic linkers is required to explain the high catalytic activity of the saturated vanadium centre in MIL-47.

This research is co-funded by Ghent University, GOA grant no. 01G00710 and Methusalem grant no. 01M00409, BELSPO in the frame of IAP 6/27 and the European Research Council (FP7(2007-2013) ERC grant no. 240483). I.M. acknowledges the IWT grant no. IWT/SB/71325. We are grateful to Steven Pijl for his experimental help with the GC-GC-TOF-MS measurement and to Jan Musschoot for the XRF analysis.

\section{Notes and references}

1 N. L. Rosi, J. Eckert, M. Eddaoudi, D. T. Vodak, J. Kim, M. O'Keeffe and O. M. Yaghi, Science, 2003, 300, 1127.

2 G. Férey, Chem. Soc. Rev., 2008, 37, 191.

3 (a) J. Lee, O. K. Farha, J. Roberts, K. A. Scheidt, S. T. Nguyen and J. T. Hupp, Chem. Soc. Rev., 2009, 38, 1450; (b) J. Perles, N. Snejko, M. Iglesias and M. A. Monge, J. Mater. Chem., 2009, 19, 6504; (c) F. Gandara, E. G. Puebla, M. Iglesias, D. M. Proserpio, N. Snejko and M. A. Monge, Chem. Mater., 2009, 21, 655; (d) M. C. Bernini, F. Gandara, M. Iglesias, N. Snejko, E. Gutierrez-Puebla, E. V. Brusau, G. E. Narda and M. A. Monge, Chem.-Eur. J., 2009, 15, 4896.

4 D. Farrusseng, S. Aguado and C. Pinel, Angew. Chem., Int. Ed., 2009, 48, 7502.

5 K. Barthelet, J. Marrot, D. Riou and G. Férey, Angew. Chem., Int. Ed., 2002, 41, 281.

6 M. J. Haanepen, A. M. ElemansMehring and J. H. C. Van Hooff, Appl. Catal., A, 1997, 152, 203.

7 E. P. Talsi, V. D. Chinakov, V. P. Babenko and K. I. Zamaraev, J. Mol. Catal., 1993, 81, 235; H. Mimoun, M. Mignard, P. Brechot and L. Saussine, J. Am. Chem. Soc., 1986, 108, 3711.

8 L. Stepovik and M. Gulenova, Russ. J. Gen. Chem., 2009, 79, 1663.

9 A. Lundin, I. Panas and E. Ahlberg, J. Phys. Chem. A, 2007, 111, 9087.

10 M. Tonigold, Y. Lu, B. Bredenkötter, B. Rieger, S. Bahnmüller, J. Hitzbleck, G. Langstein and D. Volkmer, Angew. Chem., Int. Ed., 2009, 48, 7546. 